\title{
Les femmes, le genre et les sexualités dans le Maghreb colonial (1830-1962)
}

Women, Gender and Sexualities in colonial Maghreb (1830-1962)

\section{Christelle Taraud}

\section{(2) OpenEdition}

\section{Journals}

\section{Édition électronique}

URL : https://journals.openedition.org/clio/10058

DOI : 10.4000/clio. 10058

ISSN : 1777-5299

Éditeur

Belin

\section{Édition imprimée}

Date de publication : 1 mai 2011

Pagination : 157-191

ISBN : 978-2-8107-0157-5

ISSN : $1252-7017$

Référence électronique

Christelle Taraud, "Les femmes, le genre et les sexualités dans le Maghreb colonial (1830-1962) »,

Clio. Femmes, Genre, Histoire [En ligne], 33 | 2011, mis en ligne le 01 mai 2013, consulté le 26 avril 2022.

URL : http://journals.openedition.org/clio/10058 ; DOI : https://doi.org/10.4000/clio.10058 


\section{Actualité de la recherche}

\section{Les femmes, le genre et les sexualités dans le Maghreb colonial (1830-1962)}

Jusqu'au début des années 1990, l'histoire des femmes, du genre et des sexualités en Algérie, en Tunisie et au Maroc est restée le parent pauvre d'une histoire du Maghreb, elle-même assez peu développée en France - si l'on excepte la guerre d'Algérie - au regard de l'histoire métropolitaine, européenne et occidentale stricto sensu ${ }^{1}$. Il aura fallu attendre plus de vingt ans pour que se diffuse auprès d'une nouvelle génération d'historiens et d'historiennes l'apport fondamental de l'histoire des femmes et du genre et celui des Subaltern et des Colonial Studies $^{2}$ comme le démontrent les publications, en 1999, du numéro «Femmes du Maghreb» de CLIO, HFS ${ }^{3}$ et, en 2008, d'un numéro «Colonialismes $»^{4}$ de la revue Sextant, ainsi que la naissance de deux séminaires dont le but est de faire connaitre ces nouvelles recherches $^{5}$. Dans le livre pionnier qu'elles publient en 1985, La femme

\footnotetext{
Rivet 1992.

2 Hunt, Liu \& Quataert 1997 ; Midgley 1998 ; Chaudhuri \& Roach Pierson 1998 ; Levine 2004.

3 Fine \& Leduc 1999.

4 Guiard 2008 ; Taraud 2008a ; Lauro 2008.

5 En 2008-2009, séminaire de recherches de Françoise Gaspard et Christelle Taraud, à New York University à Paris, Le genre en situation coloniale et post-coloniale ; en 2009-2010, séminaire de recherches de Brigitte Rollet à l'Institut de Londres à
} 
aux temps des colonies, Yvonne Knibielher et Régine Goutalier ${ }^{6}$ ne citent quasiment aucune étude d'histoire sur la question ${ }^{7}$ et se fondent essentiellement sur des sources secondaires sur l'Algérie coloniale: témoignages édités ${ }^{8}$, romans orientalistes et coloniaux ${ }^{9}$, premiers écrits anthropologiques et ethnographiques ${ }^{10}$ comme ceux de Germaine Tillion, de Thérèse Rivière ou de Mathéa Gaudry ${ }^{11}$ et de très rares ouvrages précurseurs comme celui d'Yvonne Turin ${ }^{12}$. De nombreux domaines sont alors ignorés et pas uniquement en France : la production anglophone est, elle aussi, insignifiante à la même époque - les départements d'histoire des universités américaines développant plutôt des travaux sur le Moyen-Orient ou sur l'Afrique sub-saharienne. Quant à la production maghrébine elle-même, du fait notamment de l'élaboration d'histoires officielles fortement marquées par le(s) nationalisme(s), elle n'est pas mieux lotie sur ces questions, souvent jugées périphériques, "coloniales» ou harâm, malgré une nette percée de l'historiographie tunisienne sous l'impulsion de Dalenda et Abdelhamid Larguèche dont l'ouvrage, Marginales en terre d'Islam, fit incontestablement date ${ }^{13}$.

Paris, Genre et cultures dans un contexte colonial et post-colonial; journée d'études, le 16 avril 2010 sur «Femme, genre et (post)colonisation?» avec Catherine CoqueryVidrovitch, Nacira Guénif-Souilamas, Emmanuel Blanchard et de jeunes chercheurs (Marie Goyon, Delphine Peiretti, Isabelle Tracol).

6 Knibielher \& Goutalier 1985.

7 Seuls sont cités Julien 1931, Girardet 1972 et Ageron 1972.

8 Amrouche 1968.

9 Bugéja 1921, Séduction orientale (1931) et Dans la tiédeur de la tente (1933) ; Henriette Célarié, Nos scurs des harems (1925), La vie mystérieuse dans les harems (1927) et Du sang et de l'amour dans les harems (1930); Lucienne Favre, Orientale (1930) et Tout l'inconnu de la casbah d'Alger (1933).

10 Les travaux de Nouvel 1919, Goichon 1927, Jouin 1932 et de Laoust-Chantréaux 1990 mériteraient d'être redécouverts.

11 Tillion 1966 ; Rivière 1995 ; Gaudry 1929, 1961.

12 Turin 1971.

13 Larguèche D. \& A. 1992. 


\section{Colonisation, peuplement et femmes européennes}

Parmi les domaines aujourd'hui en voie de développement, l'un des plus désertés à l'aube des années 1990 est celui de la place des Européennes dans la société coloniale en construction, dans les zones dites pacifiées de l'Algérie, de la Tunisie et du Maroc ${ }^{14}$. Jusqu'à la fin des années 1980, aucun livre d'histoire en France n'aborde cette question $^{15}$. Il faut attendre 1994, et la publication de l'ouvrage de Jean-Luc Einaudi sur Lisette Vincent, pour qu'un destin de femme européenne dans l'Algérie coloniale du $\mathrm{XX}^{\mathrm{e}}$ siècle soit enfin objet d'histoire ${ }^{16}$. Ce récit de vie d'une grande profondeur historique et humaine s'attachait d'abord à raconter la trajectoire exceptionnelle d'une femme singulière. De ce fait, à partir de la vie de Lisette Vincent, il était difficile d'extrapoler un quelconque modèle type de la femme «colonisatrice» en Algérie. Il en va de même du livre que Nelcya Delanoë a consacré, en 1999, à la vie et à l'œuvre de sa grandmère paternelle dans le Maroc du protectorat et qu'elle a intitulé La femme de Maragan ${ }^{17}$. En 1998, Julia Clancy-Smith et Frances Gouda coordonnent un ouvrage, Domesticating the Empire. Race, Gender and Family Life in French and Dutch Colonialism, qui ouvre des pistes de recherches intéressantes notamment sur l'Algérie des premiers temps de la colonisation (1830-1900), période moins connue que celle de la «mise en valeur» (1900-1945) ou de la décolonisation (1945-1962) ${ }^{18}$. L'ouvrage propose trois articles sur l'Algérie ${ }^{19}$, chose assez rare pour être soulignée. Il faut cependant attendre 2009 et la publication, en France, du livre de Claudine Guiard, Des Européennes en situation coloniale. Algérie 1830-193920, pour lire la première synthèse retraçant un siècle d'Algérie coloniale au prisme de l'histoire de ces Européennes qui ont largement contribué à en faire la colonie de peuplement tant espérée par l'administration métropolitaine et

14 Colonna \& Taraud 2007.

15 Robinson 1999 ne fait aucune place aux Françaises et à l'Afrique du Nord.

16 Einaudi 1994.

17 Delanoë 1999.

18 Clancy-Smith \& Gouda 1998 ; Clancy-Smith 1996a : 201-228.

19 Clancy-Smith 1998 ; Bowlan 1998 ; Fletcher 1998.

20 Guiard 2009 ; Renucci 1983 ; Dore-Audibert \& Morzelle 1997. Voir le compte rendu du livre de Claudine Guiard dans ce numéro. 
coloniale. Remarquablement documentée, mobilisant des sources nombreuses, riches et diversifiées, cette étude pionnière réhabilite avec force la nécessité d'une histoire de la colonisation qui fasse toute sa place à l'histoire des femmes et du genre. Claudine Guiard aborde de nombreuses thématiques parmi lesquelles on trouve le mariage, la fécondité et la maternité, enjeux majeurs de l'hégémonie française dans un territoire où les Européens étaient parfois supérieurs en nombre aux Français et ce, dans un monde majoritairement « indigène » globalement perçu comme menaçant et dangereux. Démarrant par une analyse circonstanciée de l'arrivée des Européennes en Algérie, de leur nombre, des raisons de leur émigration, de la relative difficulté de leur implantation, de leur installation et de leur enracinement progressifs et simultanément de la nécessité absolue de leur présence - le maréchal Bugeaud, artisan de la conquête, ne disait-il pas : " point de colonisation sans femmes »Claudine Guiard dresse ensuite une typologie fine de la condition féminine et de son évolution sur un siècle. Le chapitre «compagnes, épouses, mères » montre à quel point la colonisation en Algérie fut d'abord, après les premiers temps de la conquête où la société était plus permissive, une entreprise morale où les femmes ont été mobilisées pour construire, maintenir et entretenir un ordre colonial - sexuel et racial - qui fut un des piliers de la domination française outre-Méditerranée. Cependant, l'auteure dépasse ce constat énoncé nettement au demeurant dans ce qu'elle écrit sur l'éducation familiale méditerranéenne ${ }^{21}$, pour nous introduire dans la diversité des expériences féminines des premières migrantes ${ }^{22}$. Toute la richesse et l'intérêt de ce livre est donc de lier ce qui relève du global, de la société coloniale dans son ensemble, et de l'infiniment particulier, qui tient à la place que s'y sont faite les femmes, y compris contre les nécessités de la situation coloniale souvent au service de la domination masculine.

Cette problématique, posée par Nupur Chaudhuri et Margaret Strobel dès 1991 dans leur ouvrage Western Women and Imperialism:

21 Zagnoli \& Bréteau 1981 : 1954-2007.

22 Thème de la migration au féminin encore trop peu traité dans le cadre du Maghreb colonial. À noter cependant le récent livre de Clancy-Smith 2010. 
Complicity and Resistance ${ }^{23}$, est reprise aujourd'hui dans certains travaux historiques sur le $\mathrm{Maghreb}^{24}$ qui mettent en avant l'ambivalence des Européennes en contexte colonial, écartelées entre leur statut de colonisatrices au service de la "mission civilisatrice » et leur condition de femme. Ainsi Julia Clancy-Smith ${ }^{25}$ et Rebecca Rogers ${ }^{26}$ éclairentelles, dans leurs travaux respectifs sur les femmes et l'enseignement, des profils atypiques - Louise-Renée Millet ${ }^{27}$ à Tunis et Alix Luce ${ }^{28}$ à Alger - à la fois "complices» du projet colonial et lui résistant pourtant en ce qui concerne l'éducation des filles «indigènes». Rebecca Rogers montre bien ainsi comment $\mathrm{M}^{\mathrm{me}}$ Luce, tout en défendant un projet de colonisation culturelle des familles, et donc de la société musulmane, par le biais de l'éducation des femmes « indigènes ", s'est aussi battue, bec et ongles, pour maintenir, contre les contraintes et les contradictions de la politique éducative en Algérie et contre la volonté de l'administration coloniale française et du milieu colon, un enseignement généraliste dans l'école qu'elle fonda en 1845 à Alger. Elle conserve ce cap jusqu'à la fermeture de cette dernière, en 1861, transformée en "ouvroir» mis au service d'une politique d'éducation des filles « indigènes » par l'apprentissage.

De la même manière, on peut penser que toutes les catégories de femmes européennes qui se trouvèrent vraiment au contact des sociétés «indigènes» au Maghreb - enseignantes ${ }^{29}$, médecins et infirmières ${ }^{30}$, agricultrices, religieuses ${ }^{31}$, aventurières, exploratrices et voyageuses, artistes, prostituées - ont connu cette tension structurelle entre une appartenance à un monde européen pensé alors comme hiérarchiquement supérieur aux sociétés colonisées et leur condition de femmes qui faisaient d'elles des "grandes enfants", des mineures,

\footnotetext{
23 Chaudhuri \& Strobel 1991.

24 Lorcin 1995.

25 Clancy-Smith 2000 ; pour la Tunisie Bakalti 1990: 249-273, Kazdaghli 2000 ; pour le Maroc Knibiehler 1993 : 99-112.

26 Rogers 2009 : 39-59.

27 Catalan 1993.

28 Rebecca Rogers prépare une biographie de Mme Alix Luce.

29 Helie-Lucas 1988 et Clancy-Smith 2007 : 127-143.

30 Feyguine Abadie 1905.

31 Curtis $2006: 261-292$ et 2010.
} 
et donc des inférieures dans une société française encore régie par le code civil de 1804. Le tout est de savoir comment cette tension a pu se traduire et quelles formes éventuelles elle a pu prendre.

\section{Femmes européennes et femmes « indigènes » : une rencontre coloniale?}

Premières catégories de femmes à entrer en contact avec les sociétés «indigènes», les exploratrices et les voyageuses ${ }^{32}$. Au Maghreb, et malgré l'idée couramment répandue parmi le grand public que seules les Britanniques et les Étasuniennes exploraient et voyageaient au XIX ${ }^{\mathrm{e}}$ siècle et dans la première moitié du $\mathrm{XX}^{\mathrm{e}}$ siècle ${ }^{33}$, une plongée dans les archives et dans les ouvrages permet de retrouver de nombreuses voyageuses françaises, connues et anonymes. Ainsi, dans le livre qu'ils publient en 1994, Philippe Decraene et François Zuccarelli consacrent un chapitre entier aux femmes dans le Sahara ${ }^{34}$. Ils y (re)mettent notamment en lumière les profils atypiques d'Aurélie Picard ${ }^{35}$ et d'Isabelle Eberhardt ${ }^{36}$. Deux femmes souvent comparées ${ }^{37}$ du fait de l'exceptionnalité de leur vie à la charnière des XIX et $\mathrm{XX}^{\mathrm{e}}$ siècles, de leurs mariages mixtes avec des «Algériens» fort mal perçus à l'époque - Aurélie Picard avec Si Ahmed, cheikh de la puissante confrérie de la Tidjanyia et Isabelle Eberhardt avec le Maréchal des logis (Spahis), Slimène Enni - et des nombreux écrits sur elles publiés depuis la fin des années $1980^{38}$. Au-

32 S'ajoutent à ces voyageuses, suivant les armées de conquête, les cantinières et vivandières qui n'ont pas ou peu laissé de traces. Notons cependant les travaux de Cardoza 2010; et de Michael 2005: 21-43. De rares femmes des communautés franques vivaient dans les médinas avant la conquête coloniale (1830-1912). Il n'existe aucune étude sur elles à ma connaissance.

33 «Les récits de voyage des Françaises en Afrique du Nord ne sont pas aussi nombreux que le laisserait supposer l'implication de la France dans cette région » Hodgson 2002 : 98, 2005 et Melman 1995.

34 Decraene \& Zuccarelli 1994 ; le chapitre 5 est consacré aux femmes : 203-215.

35 Lenzini 1998.

36 Charles-Roux 1995 ; d'Eaubonne 1999 ; Delacour \& Huleu 2008.

37 Hart 1986.

38 Grâce à des archives inédites, Soazic 2009 a contesté la thèse officielle de la tentative d'assassinat d'Isabelle Eberhardt le 29 janvier 1901. 
delà de ces femmes bien connues aujourd'hui, l'histoire du voyage au féminin ${ }^{39}$ dans le Maghreb colonial se colore, dès les débuts de la conquête de l'Algérie en 1830, de destins plus obscurs et moins médiatiques comme le montre l'ouvrage, publié en 2008, de Françoise Lapeyre qui recense 70 histoires de vie de voyageuses françaises ${ }^{40}$. Ce livre passionnant ne se concentre cependant pas sur le seul Maghreb. De ce fait, certaines voyageuses françaises dans cette région ne font pas partie du panel constitué. Rebecca Rogers note ainsi l'absence de Gratia Dufaux, d'Aline Guérin ou de Louise Vallory ${ }^{41}$. Quant à Diane $W_{o o d}{ }^{42}$, elle met en avant plus particulièrement trois voyageuses françaises, Pauline de Noirfontaine, Anne Dutertre, et Augustine Girault, dont les récits, tous publiés ${ }^{43}$, apportent de nombreuses informations essentielles sur l'Algérie des années 1848-1856 tant du point de vue de la colonisation elle-même que de son impact sur les voyageuses dans la critique (plus ou moins importante selon les cas) ou dans l'adhésion totale à l'idéologie coloniale française. De même, dans un autre travail passionnant, Isabelle Ernot narre l'histoire de Madame Voisins d'Ambre, qui vécut de 1842 à 1868 en Algérie, y revint ensuite régulièrement comme "voyageuse » et fut une véritable apôtre du projet colonial et assimilateur français ${ }^{44}$. Cette femme, comme beaucoup d'autres ayant contribué par leurs actions et leurs discours à «l'aventure » coloniale, mériterait qu'on scrute de plus près son parcours, notamment par le biais d'une étude biographique circonstanciée. Les travaux menés actuellement - ceux d'Isabelle Ernot, de Diane Wood, de Barbara Hodgson, de Sarah Mills ${ }^{45}$, de Bénédicte Monicat ${ }^{46}$, de Anne-Liz Druot-Bouche ${ }^{47}-$ posent un certain nombre de questions intéressantes : celle du récit au féminin,




donc de la spécificité (ou non) du regard des femmes; celle de la publication - souvent rare car ces récits sont jugés insignifiants par les hommes de l'époque. Enfin, ces témoignages de femmes, qui oscillent souvent entre panorama «ethnographique» et vision littéraire, sont aussi captivants à décrypter du point de vue de la condition féminine. Ils permettent en effet d'élaborer une comparaison édifiante, parfois faite par les voyageuses elles-mêmes, entre statut des Européennes et des "indigènes », tout autant que de renforcer (ou au contraire de remettre en cause) certains stéréotypes et préjugés sur les femmes, mais aussi sur les hommes « indigènes ».

La chronologie des récits étudiés par Diane Wood et Isabelle Ernot permet de surcroît de croiser une autre catégorie de femmes peu connue, celle des déportées politiques dans les bagnes de l'Empire ${ }^{48}$. Trop peu de travaux historiques entremêlent encore aujourd'hui, en France, question coloniale et question sociale ${ }^{49}$. Ces deux problématiques sont pourtant intimement liées au XIX ${ }^{\mathrm{e}}$ siècle, comme dans la première moitié $\mathrm{du} \mathrm{XX}^{\mathrm{e}}$ siècle, comme le précisent tant Olivier Lecour-Grandmaison ${ }^{50}$ que Dominique Kalifa ${ }^{51}$. C'est grâce au livre qu'Odile Krakovitch a consacré, en 1990, aux femmes bagnardes que la question a été relancée en France sur le plan historiographique ${ }^{52}$. On constate cependant que, malgré le travail réalisé par cette dernière - notamment pour inventorier et référencer les archives sur la question, les études restent rares, y compris pour les profils exceptionnels de la déportation au féminin. Seul un livre déjà ancien a ainsi été consacré à la transportation en Algérie de Pauline Roland, après le coup d'État de Louis Napoléon Bonaparte en 1851. Encore faut-il préciser qu'il s'agit du témoignage de Pauline Roland elle-même, annoté et publié par Fernand Rude ${ }^{53}$. Louise Michel, la grande communarde de 1871, est en revanche mieux lotie ; cependant elle n'est pas déportée en Algérie, mais en Nouvelle-

\footnotetext{
48 Taraud 2009a : 17-25.

49 Hefferman 1989 : 377-403.

50 Lecour-Grandmaison 2005.

51 Kalifa 2009.

52 Krakovitch 1998 [1990].

53 Textes de Pauline Roland, Arthur Ranc, Gaspard Rouffet par Rude 1981.
} 
Calédonie ${ }^{54}$. À noter toutefois le livre que consacre, en 2007, Clotilde Chauvin à la série de conférences que Louise Michel donne, en compagnie d'Ernest Girault, en octobre et décembre 1904 en Algérie, dénonçant très vigoureusement les méfaits de la colonisation ${ }^{55}$ - méfaits qu'elle avait déjà vus à l'œuvre lors de la grande révolte canaque de 1878. C'est donc tout un pan de l'histoire des femmes et $\mathrm{du}$ genre qui se trouve ici en attente de nouveaux travaux ${ }^{56}$ notamment en ce qui concerne les femmes « indigènes » transportées ou déportées seules ou avec leurs époux dans d'autres parties de l'Empire ou en métropole, thème qui reste un des angles morts de la recherche. À ma connaissance, la seule étude en cours sur des femmes « indigènes" déportées est celle d'Amel Chaouati sur La situation des femmes et des enfants de la famille et de la suite de l'Émir Abdelkader pendant leur emprisonnement au château d'Amboise de 1848 à 185257. Amel Chaouati retrace ce que fut ce séjour pour des femmes nomades et musulmanes de l'Ouest algérien catapultées dans une terre étrangère et «infidèle» au climat difficile. Ne sortant pas, ne parlant que l'arabe, soumises à des conditions d'emprisonnement extrêmement dures, ces femmes développent des maladies telles la mélancolie ou la langueur... et meurent ainsi que leurs enfants. Les 25 tombes du château d'Amboise, comme le signale à juste titre Amel Chaouati, sont encore là pour témoigner de cette histoire douloureuse ${ }^{58}$.

À l'exception notable des voyageuses, peu de Françaises ou d'Européennes semblent avoir écrit, de 1830 à la veille de la Première Guerre mondiale, sur les femmes «indigènes » en Afrique du Nord. Peu d'écrivaines, hormis Isabelle Eberhadt ${ }^{59}$, mais aussi peu de journalistes, à l'exception de Paule Mink, et de militantes féministes,

54 Michel 1885 ; d'Eaubonne 1985 et Cappella 2005.

55 Chauvin 2007.

56 Voir pour l'Australie dans l'Empire britannique Selzer 1994 ; Oxley 1996 ; Damousi 1997 ; Swiss 2010 ; McIntyre 2010 ; Kassis 2010.

57 Chaouati, publication en cours.

58 Que sait-on des femmes kabyles déportées avec leur époux à la suite de la grande révolte du Bachaga Mokrani de 1871 ? Voir Lallaoui 1999.

59 J'aime particulièrement dans l'œuvre d'Isabelle Eberhadt la nouvelle «Sous le joug » publiée en octobre 1902 ; Zeys 1908. 
travaillent alors en Algérie ${ }^{60}$. Hubertine Auclert est à contre-courant de ce mouvement général. Il est vrai que de ses quatre années passées en Algérie, elle publie en 1900 un témoignage poignant et accablant, Les femmes arabes en Algérie, sur la condition des femmes "indigènes » soumises à la double domination masculine et coloniale. Ce travail que l'on peut considérer comme extraordinaire a donné lieu à un certain nombre de recherches depuis les années 1990. Ainsi, Carole Wiart lui a consacré son mémoire de maitrise en 1997, tandis que Julia Clancy-Smith en 1998, Edith Taïeb en 2007 et Carolyn Eichner en 2009 écrivaient, dans des registres différents, sur la singularité et la radicalité de son propos ${ }^{61}$. Autre femme atypique, Elissa Rhaïs, juive francisée, dont les romans ${ }^{62}$ ont un grand succès dans les années 1920 tant en Algérie qu'en métropole ${ }^{63}$. À partir de cette date, la Grande Guerre faisant ici incontestablement césure, plusieurs écrivaines françaises traitent régulièrement de la condition des femmes « indigènes» dans leurs ouvrages. Parmi ces dernières, Aline Reveillaud de Lens ${ }^{64}$, Marie Bugéja ${ }^{65}$, Henriette Célarié ${ }^{66}$ ou Lucienne Favre $^{67}$. Aux titres souvent racoleurs, les ouvrages de ces femmes écrivains et/ou journalistes, ont donné lieu à trop peu de recherches spécifiques, si l'on excepte celles de Patricia Lorcin ${ }^{68}$, de Sakina Messaâdi ${ }^{69}$ et de Jeanne Bowlan ${ }^{70}$. Ils traduisent cependant une prise de conscience qui touche, dans le même temps, le milieu féministe français. La question des femmes aux colonies fait d'ailleurs l'objet

60 Mink $1886: 12-14$.

61 Wiart 1997 ; Clancy-Smith 1998 : 154-174 ; Taïeb 2001 : 271-281 ; Eichner 2009 : 63-84.

62 Saada la Marocaine, Paris, Plon, 1919 [réédité par les Éditions Bouchene en 2002], et Le café chantant, Paris, Plon, 1920 [idem 2003].

63 Sur Elissa Rhaï, voir Dejeux 1984 : 47-79 ; Balhoul 1985 : 275-281 ; Tabet 1982 ; téléfilm d'Otmezguine 1993.

64 Reveillaud de Lens 1922, 1925, 2006.

65 Bugéja 1919, 1921, 1931 et 1933.

66 Célarié 1925, 1927 et 1930.

67 Favre 1930 et 1939.

68 Lorcin 2002 : 163-184, $2003: 108-130,2004: 45-61$.

69 Messaâdi 1990.

70 Bowlan 1998 : 175-192. 
des États généraux du féminisme réunis dans le cadre de l'exposition coloniale internationale de 1931, comme l'explique Régine Goutalier dans un article précurseur publié en 198971. Même si les débats de l'époque restent empreints d'un certain «maternalisme colonial» - lui-même plus ou moins teinté de l'idéologie racialiste de l'époque la spécificité de la question des femmes «indigènes» est abordée concrètement et collectivement pour la première fois comme le montre Sara Kimble pour l'Algérie ${ }^{72}$. Le problème des rapports entre colonisation et féminisme européen et entre militantes européennes et femmes "indigènes »- souvent prises en tenaille, du fait de la situation coloniale, entre leur désir d'émancipation et leur appartenance à des «traditions» sociales et religieuses locales, mériterait d'être mieux traité, même si des travaux récents comme ceux de Julia Clancy-Smith, de Deborah Cherry ${ }^{73}$, de Sakina Messaâdi ${ }^{74}$ ou de Jennifer Boittin ${ }^{75}$ ouvrent la voie à des recherches nouvelles. De même, il serait intéressant de regarder de près la presse féminine et féministe en métropole et au Maghreb. En Algérie par exemple, deux journaux, La Femme algérienne et Femmes de demain, sont créés dans les années 1930. Le second, animé par Lucienne JeanDarrouy - qui est membre du bureau de l'Union des Françaises pour le suffrage des femmes à Alger - sera le relais efficace des grandes questions qui se posent à l'Algérie et à la France de l'époque : projet Blum-Viollette, suffrage des femmes en métropole, condition des musulmanes ${ }^{76}$.

Cependant, le véritable point noir de l'historiographie contemporaine ne concerne pas tant le féminisme européen dans ses liens, ambigus et conflictuels, avec les mondes féminins autochtones - mondes qui se trouvent, à partir des années 1930, au cœur d'un combat idéologique qui a tout à voir avec le maintien de l'hégémonie française au Maghreb comme le démontrent clairement les débats et

\footnotetext{
71 Goutalier 1989 : 266-254. Voir Legrand-Falco 1931.

72 Kimble 2006 : 109-128.

73 Cherry 1996.

74 Messaâdi 2002.

75 Boittin 2010a : 131-150.

76 Guiard, dossier « Presses et Colonies », Revne de la BnF, 2011.
} 
les polémiques autour des législations de 1931 et de 195977 , du droit de vote des femmes algériennes, tunisiennes et marocaines après $1945^{78}$ et de la bataille du voile en $1958^{79}$ - mais plutôt l'histoire de la création de mouvements féminins ou féministes chez les femmes « indigènes » elles-mêmes. Ces mouvements ne peuvent être compris que dans leur interaction, souvent structurelle, avec le réformisme musulman d'une part et/ou le nationalisme d'autre part. Ce qui explique que les études, toujours rares sur ces questions, soient marquées depuis la publication en 1994 du livre de Zakya Daoud, Féminisme et politique au Maghreb80, par cette problématique tant en Algérie $^{81}$, en Tunisie ${ }^{82}$ qu'au Maroc ${ }^{83}$. Ils doivent aussi être perçus dans la tension, inhérente à la situation coloniale, entre émancipation et «tradition ». Ainsi, entre 1924 et 1929, Manoubia Ouertani et Habiba Menchari, deux des plus grandes féministes tunisiennes de la période - qui n'ont pas fait l'objet, à ma connaissance, de biographies historiques - sont-elles accusées d'être inféodées à la France après qu'elles se soient dévoilées en public et exprimées, de manière renouvelée, sur la question de l'analphabétisme des femmes, sur leur claustration, sur les mariages forcés dont elles sont les victimes, sur les répudiations. Cette tension, dont les manifestations sont multiples, devrait donner lieu, dans l'avenir, à de nombreuses études au Maghreb mais aussi dans l'ensemble du monde musulman. Ainsi, il manque aujourd'hui une synthèse historique sur la création et le développement de féminismes orientaux dont les foyers les plus importants furent, dès la fin du XIXe siècle, la Turquie, l’Égypte, la Syrie et la Tunisie.

77 Sur la réforme en 1931 du statut de la femme kabyle et sur celle du mariage en 1959 (tentative de lier droit islamique, coutumes et code civil français) voir Charrad 2001.

78 Le problème de l'application du droit de vote des femmes aux territoires d'Afrique du Nord n'a pas donné lieu à un travail historique spécifique.

79 Sheppard $2004:$ : 134-141.

80 Daoud 1994.

81 Sai 1984 ; Lazreg 1994 ; Lalami 2008 : 16-27.

82 Marzouki 1993 ; Bakalti 1996 ; Sraieb 1999 : 75-92.

83 Benadada 1999 : 67-73 ; Taraud 2007 : 115-125. 


\section{L'orientalisme recontextualisé}

Notons d'abord le peu de travaux menés par les historiens et les historiennes du Maghreb contemporain, tant en Europe, en Amérique du Nord qu'au Maghreb, sur les femmes «indigènes » en contexte colonial. Confrontés à ce qu'il faut bien appeler un véritable "désert historiographique ${ }^{84}$ à l'aube des années 1980 , ce sont les sociologues qui prennent d'abord le relais de l'anthropologie et de l'ethnographie des années 1930-1960: des travaux menés par Fadela M'Rabet, en 1967, Les Algériennes ${ }^{85}$, et par Nefissa Zerdoumi, en 1970, Enfants d'bier, enfants d'aujourd'bui. L'éducation de l'enfant en milieu traditionnel algérien ${ }^{86}$, aux écrits de Fatima Mernissi dans les années 1980 et $1990^{87}$. En 2000, Dalenda Larguèche dirige un ouvrage collectif, Histoire des femmes an Maghreb. Culture matérielle et vie quotidienne $^{88}$ qui amorce un regain d'intérêt de l'histoire pour les questions ayant trait aux femmes et au genre. Malgré cela, force est de constater que de nombreux domaines, pourtant essentiels à cette histoire, restent encore quasiment inexplorés : le religieux ${ }^{89}$, y compris dans les liens que les femmes des trois religions du Livre peuvent entretenir entre elles au Maghreb ; les communautés au fémininin ${ }^{90}$; le politique et les espaces publics; le travail, salarié ou non; la santé, l'hygiène et la reproduction ${ }^{91}$; les familles et tout ce qui les constitue (notamment les codes du statut personnel et leur évolution au cours

84 Du fait du désintérêt pour l'histoire de la colonisation en France et au Maghreb après les indépendances; de la concentration des universités américaines sur d'autres aires comme le Moyen-Orient et l'Afrique sub-saharienne; de l'illégitimité de ces questions en France et en Europe et par voie de conséquence d'une absence chronique d'institutionnalisation...

85 M'Rabet 1967.

86 Zerdoumi 1970.

87 Mernissi 1983, 1987, 1990 et 2001.

88 Larguèche 2000 ; Sambron 2009.

89 Sur l'islam et sur le judaïsme au féminin pendant la période coloniale, aucune étude historique récente à ma connaissance à l'exception de l'article de Schreier 2007 : 77-103. Voir aussi sur les conversions au christianisme dans la société kabyle Slimani Dirèche 2004.

90 À ma connaissance, aucune étude ne traite spécifiquement des femmes juives ou des femmes noires en Algérie, en Tunisie et au Maroc.

91 Colin 1998. 
de la période coloniale $)^{92}$; la construction des identités de genre... Si l'on excepte la question de l'éducation et de la scolarisation, plutôt bien traitée comme nous l'avons vu au début de cet article, seuls trois domaines sont bien présents dans l'historiographie récente: les représentations de l'Autre, la sexualité et la guerre d'Algérie.

On ne s'étonnera pas en effet qu'à la suite de la publication par Edward Saïd, en 1978, de Orientalism - traduit en français au Seuil, dès 1980, sous le titre L'Orientalisme. L'Orient créé par l'Occident- se soit développé un vaste courant liant les apports théoriques de ce livre pionnier, mais qui parle cependant fort peu des femmes et du genre, avec ceux des Women et des Gender Studies. Aux États-Unis, les études se multiplient ainsi, dès le début des années 1990, avec des travaux comme ceux de Linda Nochlin ${ }^{93}$, de Reina Lewis ${ }^{94}$, de Meyda Yeyenoglu ${ }^{95}$, et de Yaël Simpson Fletcher ${ }^{96}$. En France, le livre dirigé par Marie-Elise Palmier-Chatelain et Pauline Lavagne d'Ortigue, L'Orient des femmes, fait incontestablement date par sa qualité et son érudition ${ }^{97}$. L'étude des représentations ${ }^{98}$, dans la peinture ${ }^{99}$, la photographie $^{100}$, les cartes postales ${ }^{101}$, le cinéma ${ }^{102}$ ou la littérature ${ }^{103}$, produit aussi des travaux nouveaux de grande tenue. Ces derniers permettent à certaines thématiques, comme le harem par exemple, de redonner souffle à une historiographie dont la voie avait été ouverte par l'ouvrage désormais classique d'Alain Grosrichard ${ }^{104}$. Dans la

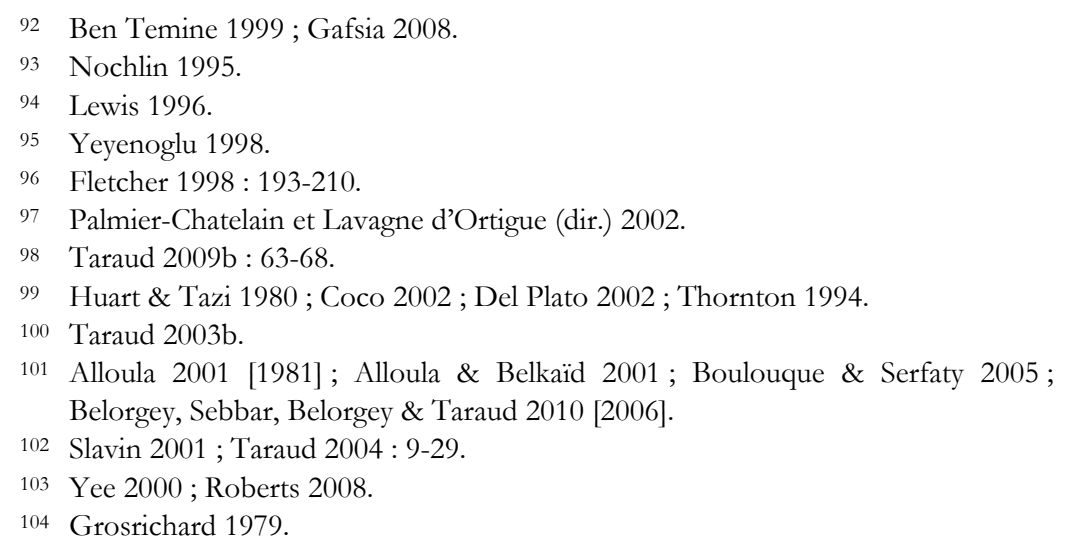


foulée de ces grandes études sur les représentations du harem ${ }^{105}$, vont en effet être (re)publiés des témoignages de «femmes au harem», comme ceux de Lady Mary Montagu ${ }^{106}$ et de Leila Hanoum ${ }^{107}$ et de nouveaux ouvrages sur l'institution elle-même. Ainsi, les travaux menés aux États-Unis par Leslie Pierce ${ }^{108}$ et ceux produits en France par Jocelyne Dakhlia ${ }^{109}$ ont permis de revivifier le champ ${ }^{110}$ tant du point de vue du rôle et de la place des femmes à l'intérieur du harem, que de la question des rapports de genre qui y sont à l'œuvre. Le harem n'est donc plus seulement perçu et pensé comme un lieu d'atonie et d'enfermement des femmes - ce qu'il a trop longtemps été dans une certaine historiographie fortement inspirée des stéréotypes orientalistes - mais aussi comme un espace de pouvoir féminin (ou s'exercent par exemple des violences entre femmes); de hiérarchie entre individus libres et esclaves, entre hommes et femmes (où la mère du sultan ou du prince a des pouvoirs bien plus considérables que de très nombreux hommes), entre femmes elles-mêmes (domestiques, odalisques, favorites...). Lieu de sexualité licite et illicite (les gitons du prince, le «lesbianisme» des femmes, l'adultère...), le harem apparait, dans ces nouvelles approches, comme un espace où les genres se côtoient, se frôlent, s'entrechoquent et peuvent même, parfois, s'inverser comme dans le cas des eunuques ${ }^{111}$. Comme l'historiographie du harem, celle de l'eunuchisme à l'époque moderne et contemporaine ${ }^{112}$ est, elle aussi, surtout centrée sur le cœur de l'Empire ottoman, Istanbul, et fait, malheureusement, trop peu de place au Maghreb ${ }^{113}$. Ajoutons à cela que la question connexe de l'esclavage des femmes est elle aussi peu

\footnotetext{
105 Croutier 1990.

106 Montagu 2001.

107 Hanoum 2000.

108 Pierce 1993.

109 Dakhlia 1999: 37-55, 2007a : 61-88. Séminaire de recherches à l’EHESS sur Harems et despotisme.

110 Booth 2011.

111 Dakhlia 2007 : 1097-1120.

112 Hataway 1992 : 141-158, 1994 : 293-317 et 2005.

113 Dakhlia 2000.
} 
traitée ${ }^{114}$. Notons cependant le beau livre de Mohammed Ennaji, Soldats, domestiques et concubines. L'esclavage an Maroc an XIXe siècle, publié en 1994115.

\section{Sexualités en situation coloniale}

La question de la sexualité en contexte proprement colonial ${ }^{116}$ est, en revanche, mieux lotie ${ }^{117}$. Sous l'impulsion des travaux fondateurs de Michel Foucault et de Jean-Louis Flandrin ${ }^{118}$ sur la sexualité en Occident, croisés avec ceux non moins pionniers de G.H. Bousquet, L'éthique sexuelle de l'islam ${ }^{119}$, d'Abdelwahab Boudhiba, La sexualité en Islam $^{120}$, de Margaret Strobel ${ }^{121}$ et de Ann Laura Stoler ${ }^{122}$, c'est tout un pan de la recherche qui s'est développé de la fin des années 1980 au début des années 1990. D’abord timide, plutôt centrée sur l'histoire des marginalités et des minorités comme le montrent les ouvrages de Dalenda et Abdelhamid Larguèche ${ }^{123}$, de Fanny Colonna ${ }^{124}$, de Jacques Alexandropoulos et Patrick Cabanel ${ }^{125}$, la recherche en ce domaine s'est ensuite affirmée dans des directions diverses : mixité sexuelle ${ }^{126}$ et prostitution ${ }^{127}$; enfants métis ${ }^{128}$; homosexualité ${ }^{129}$. Les questions sexuelles, comme le démontrent clairement des ouvrages

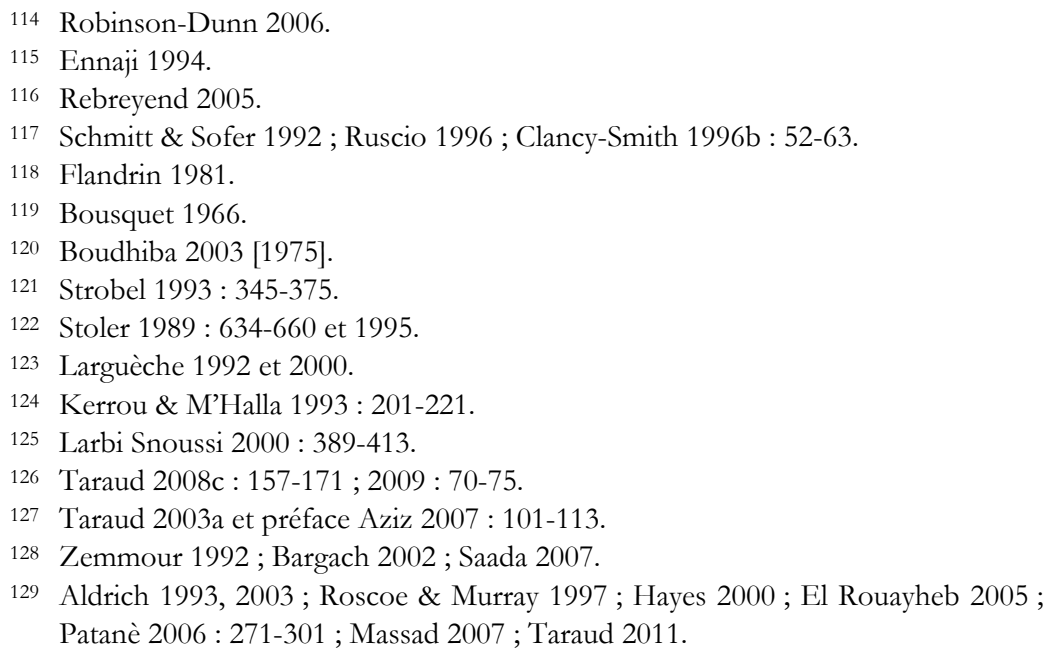


comme La prostitution coloniale de Christelle Taraud ${ }^{130}$ ou Colonialism and Homosexuality de Robert Aldrich ${ }^{131}$, sont en effet au cœur de la politique coloniale française, qu'il s'agisse de lutter contre la multiplication des couples mixtes franco-maghrébins - par peur d'une contamination religieuse et/ou raciale - de nier ou d'occulter l'existence d'enfants métis, pourtant produits de cette «rencontre coloniale ", ou d'organiser un marché du sexe, tant en milieu civil (maisons de tolérance et quartiers réservés) qu'en milieu militaire (Bordels Militaires de Campagne) dans le cadre d'un réglementarisme colonial mis en place en Algérie, en Tunisie et au Maroc dès le début des conquêtes militaires dans les zones «pacifiées» par l'armée d'Afrique. Pensées dès lors comme des «paradis sexuels» où toutes les combinaisons sont possibles, les colonies deviennent des espaces de dilatation du désir de l'Autre, désir qui s'exerce dans un mélange complexe de fascination et de répulsion du fait des contradictions de l'idéologie coloniale en ce domaine, et, simultanément, des lieux de «tourisme sexuel »- tant hétérosexuel qu'homosexuel ${ }^{132}-$ dans des villes phares comme Alger, Oran, Casablanca, Tanger ou Tunis ${ }^{133}$. À la question du «tourisme sexuel », dont l'histoire reste globalement à faire en Afrique du Nord, se superpose donc celle de l'homosocialité, de l'homoérotisme ${ }^{134} \mathrm{ou} /$ et de l'homosexualité. Au-delà de cet aspect, l'histoire des relations - sexuelles ou non - entre hommes dans le Maghreb colonial invite à une réflexion plus large sur la construction de la virilité et de la masculinité, en lien avec la question raciale et nationale, tant dans l'Empire ${ }^{135}$ qu'en métropole ${ }^{136}$. À regarder de près l'installation de la France au Maghreb, on est en effet frappé par le caractère éminemment viril de l'entreprise, tant dans la production de discours très structurés et normatifs sur la masculinité137 de

130 Taraud 2003a.

131 Aldrich 2003.

132 Taraud, à paraître.

133 Finlayson 1993.

134 Dakhlia 2007b : 1097-1120.

135 Benali 1998 : 147-160 ; Benslama \& Tazi 2004 ; Taraud 2011 et à paraître.

136 Blanchard 2008 : 209-224 ; Camiscioli 2009.

137 En 1861, dans la neuvième édition du Bescherelle - Dictionnaire national on Dictionnaire universel de la langue française - on trouve la définition suivante de la 
l'Empire - discours dans lesquels la question de la virilité138 a toute sa place - que dans l'élaboration de catégories qui organisent et façonnent en miroir les rapports entre les Français et les Autres perçus collectivement dans une altérité d'autant plus radicale qu'elle est un enjeu essentiel des conflits de conquête et des politiques de «mise en valeur» qui leur succèdent. Dès lors, s'intéresser à la virilité et à la masculinité, c'est autant interroger la colonisation virile elle-même (dans sa version militaire et civile) que questionner la manière dont la politique coloniale a organisé une véritable dévirilisation et domestication des «indigènes ». Faire l'histoire de ce processus permet, de surcroît, d'éclairer sous un jour nouveau la question des nationalismes au Maghreb et celle des décolonisations.

\section{Femmes, hommes et guerre en contexte colonial}

Jusqu'à une date récente, peu d'études ont pris en compte la dimension du genre dans les guerres de conquête et de pacification au Maghreb, y compris en Algérie. Soulignons aussi que les conflits comme la guerre du Rif dans les années 1920, n'ont jamais fait l'objet d'une histoire spécifique, et encore moins d'une histoire des femmes et du genre. À l'exception notable des récents travaux de Jean-Yves Le Naour et d'Armelle Mabon sur l'implication des troupes coloniales dans les deux conflits mondiaux en Europe ${ }^{139}$ et de ceux de Nelcya Delanoë sur leur rôle dans le maintien de l'ordre colonial dans l'Empire colonial français ${ }^{140}$, seule la guerre d'Algérie possède une historiographie conséquente ${ }^{141}$. Dans cette optique, le premier livre marquant est Les femmes algériennes dans la guerre, de Djamila Amrane ${ }^{142}$.

masculinité : «Ce qui accompagne le penchant de l'homme à s'approprier tout ce qui annonce de la grandeur, de la force, de la supériorité », Corbin 2007 : 7.

138 "Se viriliser, c'est prendre le ton et les manières d'un homme ", c'est-à-dire affirmer cette puissance ou capacité d'engendrer que celui qui est possesseur du "membre viril" doit en toute occasion, manifester par des signes clairs », Corbin, ibid.: 7. Ce qui est valable pour l'individu peut être étendu au collectif, notamment en situation coloniale.

139 Le Naour 2004, 2006 ; Mabon 2010.

140 Delanoë 2002.

141 Jauffret \& Ageron 2003.

142 Amrane 1991. 
À sa suite, un certain nombre d'études comme celles de Diane Sambron ${ }^{143}$ et de Ryme Seferdjeli144 ont permis de mieux comprendre l'implication des femmes algériennes dans la guerre de Libération femmes anonymes ${ }^{145}$ ou célèbres ${ }^{146}$ - les différents rôles qu'elles ont $\mathrm{pu}$ y jouer: militantes civiles (moussebilates) ou combattantes (mondjabidates ou fidayates), les résistances qu'elles ont pu rencontrer de la part des hommes en général et des cadres et militants du FLN/ALN en particulier, mais aussi la manière dont elles ont pu être utilisées dans la propagande coloniale, y compris par l'armée française ${ }^{147}$. D'abord centrée, du fait du succès du film La bataille d'Alger de Gillo Pontecorvo (sorti sur les écrans en France en 1966), sur les grandes figures au féminin de la guerre, notamment sur les poseuses de bombes de la bataille d'Alger de 1957148, l'historiographie prend ensuite en compte la question des femmes algériennes victimes du conflit ${ }^{149}$. Femmes arrêtées, battues, torturées, violées, parfois aussi assassinées. À l'époque de la guerre d'Algérie, Gisèle Halimi et Simone de Beauvoir s'étaient faites l'écho du calvaire de Djamila Boupacha ${ }^{150}$. Aujourd'hui, les travaux de Raphaëlle Branche sur la torture et le viol ${ }^{151}$, mais aussi sur la virilité et la masculinité152, permettent de regarder la guerre d'Algérie selon de nouvelles perspectives $^{153}$. Perspectives travaillées, dans des directions différentes, par d'autres historiens comme Todd Sheppard ${ }^{154}$ et Claire Mauss-Copeaux ${ }^{155}$. À cela s'ajoute un certain nombre d'ouvrages sur




des catégories de femmes moins abordées par l'historiographie de la guerre : femmes et filles de harkis mises en lumière par Fatima Besnaci-Lancou ${ }^{156}$ et femmes européennes impliquées dans la guerre ou simplement la subissant ${ }^{157}$. De ce passé lié à la guerre émergent encore d'autres figures telle celle de Louisette Ighilariz ${ }^{158}$. Cette dernière a d'ailleurs raconté son histoire dans un livre, Algérienne, publié en $2001^{159}$.

Ces figures d'un passé colonial mal assumé - comme le montre, entre autre chose, la récente polémique sur l'enseignement de l'histoire de la colonisation en France dont on sait qu'elle concernait surtout l'Afrique du Nord et surtout l'Algérie - nous oblige à un devoir d'histoire qui donne véritablement leur place aux femmes et au genre dans le Maghreb colonial. Sans prétendre que cette dimension doive être exclusive dans l'élaboration d'une nouvelle histoire de cette région centrale pour l'Empire français - il faut au contraire varier les approches et les catégories d'analyse ${ }^{160}$ - les études menées depuis le début des années 1990 soulignent cependant à quel point cette dernière peut être stimulante et enrichissante pour l'histoire globale du Maghreb. Si de très nombreux aspects de la question restent à défricher, d'autres fonctionnent comme des locomotives dans un champ en pleine expansion. Reste à combler les vides... Ce sera le rôle de la nouvelle recherche en histoire dans les années à venir.

\section{Bibliographie}

AgERON Robert, 1972, "Gambetta et la reprise de l'expansion française », Revue française d'bistoire d'Outre-mer, t. LIX, 215.

AlDrich Robert, 1993, The Seduction of the Mediterranean: Writing, Art and Homosexual Fantasy, Londres/New York, Routledge.

_, 2003, Colonialism and Homosexuality, Londres/New York, Routledge.

\footnotetext{
156 Besnaci-Lancou 2006.

157 Dore-Audibert 1995 ; Foche-Duval 2007 ; Jauneau 2008 : 71-85 ; Plisson 1972.

158 Témoin pendant le procès Aussarès, elle fut torturée et violée pendant la guerre.

159 Ighilahriz 2001.

160 Taraud 2008c.
} 
Alloula Malek, 2001 [1981], Le Harem colonial, Paris, Slatkine.

Alloula Malek \& Leyla BelkaïD, 2001, Belles Algériennes de Geiser, costumes, parures et bijoux, Paris, Éditions Marval.

Amrane Djamila, 1991, Femmes algériennes dans la guerre, Paris, Plon.

—, 1993, Femmes au combat : la guerre d'Algérie (1954-1962), Alger, Éditions Rhama.

—, 1999, «Femmes dans la guerre d'Algérie. Entretiens avec Fatma Baïchi», in Agnès Fine \& Claudine Leduc (dir.), «Femmes du Maghreb », CLIO. HFS, 9, p. 223-246.

Amrouche Aït Mansour Fadhma, 2000 [1 re éd. 1968, réed. 1991], Histoire de ma vie, Paris, Maspero.

AzIz Germaine, 2007, Les chambres closes, Paris, Nouveau Monde Éditions.

BAKALTI Souad, 1990, "L'enseignement féminin dans le primaire au temps de la Tunisie coloniale », Revue de l'Institut des Belles Lettres Arabes, 53/166, p. 249-273.

-, 1996, La femme tunisienne au temps de la colonisation, 1881-1956, Paris, L'Harmattan.

Balhoul Joëlle, 1985, «Elissa Rhaïs », in Albert MEMMI (dir.), Écrivains francophones du Maghreb, anthologie, Paris, Seghers, p. 275-281.

Bard Christine \& Christelle Taraud (dir.), 2003, «ProstituéEs », Clio. HFS, 17, Toulouse, Presses universitaires du Mirail.

BARGaCH Djamila, 2002, Orphans of Islam. Family, Abandomnent and select adoption, Rowwan \& Littlefield.

Belorgey Jean-Michel, Sebbar Leila \& Christelle Taraud, 2010 [2006], Femmes d'Afrique du Nord. Cartes postales (1885-1930), Paris, Bleu Autour.

BENADADA Assia, 1999, "Les femmes dans le mouvement nationaliste marocain ", in Agnès Fine \& Claudine LeduC (dir.), «Femmes du Maghreb», CLIO. HFS, 9, Toulouse, PUM, p. 67-73.

BENALI Abdelkader, 1998, «Le maghrébin efféminé : le cinéma colonial ou l'autopsie d'un fantasme politique », Cabiers intersignes, 11-12, printemps, p. 147-160.

Benslama Fethi \& Nadia TAZI (dir.), 2004, La virilité en Islam, La tour d'Aigues, Éditions de l'Aube.

BESNACI-LANCOU Fatima, 2005, Fille de harki : Le bouleversant témoignage d'une enfant de la guerre d'Algérie, Paris, L'Atelier.

—, 2006, Nos mères, paroles blessées : une autre histoire de barkis, Paris, Soleil-Zellige.

BLANCHARD Emmanuel, 2008, «Le mauvais genre des Algériens. Des hommes sans femme face au virilisme policier dans le Paris d'après-guerre », in Carmen Bernand, Capucine Boidin \& Luc Capdevila (dir.), "Amériques métisses ", CLIO. HFS, 27, Toulouse, Presses universitaires du Mirail, p. 209-224. 
BOITTIN Jennifer, 2010a, «Feminist mediations of the exotic: French Algeria, Morocco and Tunisia, 1921-1939», Gender \& history, 22/1, p. 131-150.

—, 2010b, Colonial Metropolis: The Urban Grounds of Anti-Imperialism and Feminism in Interwar Paris, Nebraska, University of Nebraska Press.

Booth Marilyn, 2010, Harem Histories: Envisioning Places and Living Spaces, Durham/Londres, Duke University Press.

BoudHiBA Abdelwahab, 2003 [1975], La sexualité en islam, Paris, Presses universitaires de France.

Boulouque Clémence \& Nicole S. Serfaty, 2005, Juives d'Afrique du Nord. Cartes postales (1885-1930), Paris, Bleu Autour.

Bousquet Georges Henri, 1966, L'éthique sexuelle de l'islam, Paris, Maisonneuve et Larose.

Bowlan Jeanne M., 1998, « Civilizing Gender Relations in Algeria: The Paradoxical Case of Marie Bugeja, 1919-1939 », in Julia ClanCY-Smith \& Frances GoudA (eds), Domesticating the Empire, Charlottesville/Londres, University Press of Virginia, p. 175-192.

BRAC DE LA PERRIÈRE Caroline, 1987, Derrière les béros. Les employées de maison musulmanes au service des Européens pendant la guerre d'Algérie, 1954-1962, Paris, L'Harmattan.

BRANCHE Raphaëlle, 2001, La Torture et l'armée pendant la guerre d'Algérie, 1954-1962, Paris, Gallimard.

—, 2002a, «Des viols pendant la guerre d'Algérie », Vingtième Siècle, Revue d’histoire, 75, p. 123-132.

—, 2002b, "Être soldat en Algérie face à un ennemi de l'autre sexe », Annales de Bretagne et des Pays de l'Ouest, 109/2, p. 143-150.

—, 2004, «La masculinité à l'épreuve de la guerre sans nom », in Luc CAPDEVILA \& Dominique Godineau (dir.), « Armées », CLIO. HFS, 20, p. 111-122.

_ 2009, «Le sexe, le genre et la parole : quand une femme interroge des hommes sur les violences infligées », Françoise ThÉBAud \& Geneviève DermenjIAN (dir.), Quand les femmes témoignent: histoire orale, bistoire des femmes, mémoire des femmes, Paris, Publisud, p. 217-226.

—, 2010, L'embuscade de Palestro. Algérie 1956, Paris, Armand Colin.

BugÉja Marie, 1919, A travers l'Algérie, impressions sur la femme musulmane, Alger, Imprimerie Algérienne.

—, 1921, Nos sæurs musulmanes.

—, 1931, Séduction orientale. 
—, 1933, Dans la tiédeur de la tente.

CAMisCIOLI Elisa, 2009, Reproducing the French Race. Immigration, Intimacy, and Embodiment in the Early Twentieth Century, Durham \& Londres, Duke University Press.

Cappella Emilie, 2005, Louise Michel : exil en Nouvelle-Calédonie, Paris, Magellan \& Compagnie.

CARDoza Thomas, 2010, Intrepid Women: Cantinières and Vivandières of the French Army, Indiana, Indiana University Press.

Catalan Marianne, 1993, "Scolarité féminine et émancipation en pays arabomusulman : l'école Louise-René Millet de Tunis, 1900-1956 », mémoire de DEA, Université Aix-Marseille.

CÉLARIÉ Henriette, 1925, Nos sæurs des harems.

—, 1927, La vie mystérieuse dans les harems.

—, 1930, Du sang et de l'amour dans les harems, Paris, Hachette.

Chaouati Amel, La situation des femmes et des enfants de la famille et de la suite de l'Émir Abdelkader pendant leur emprisonnement au château d'Amboise de 1848 à 1852 (à paraitre).

Charrad Mounira M., 2001, States and Women's Rights: The Making of Postcolonial Tunisia, Algeria \& Morocco, Berkeley, California University Press.

CHARles-Roux Edmonde, 1991, Un désir d'Orient : jeunesse d'Isabelle Eberhardt, Paris, LGF.

—, 1995, Nomade j'étais. Les années africaines d'Isabelle Eberhardt, Paris, Grasset.

Chaudhuri Nupur \& Ruth RoAch Pierson (eds), 1998, Nation, Empire, Colony: Historicizing Gender and Race, Bloomington, Indiana University Press.

Chaudhuri Nupur \& Margaret STROBEL (eds), 1991, Western Women and Imperialism: Complicity and Resistance, Indiana, Indiana University Press.

Chauvin Clotilde, 2007, Louise Michel en Algérie : la tournée de conférences de Louise Michel et Ernest Girault en Algérie, octobre-décembre 1904, Saint-Georges-d'Oléron, Éditions Libertaires.

CHERry Deborah, 1996, «Shuttling and Soul-Making: Tracing the Links between Algeria and Egalitarism Feminism in the 1850s », in Shearer WeST (ed.), The Victorians and Race, Aldershot, Scholar Press.

Clancy-Smith Julia, 1996a, "The Colonial Gaze: Sex and Gender in the Discourses of French North Africa », in Carl L. Brown \& Mathew S. Gordon (eds), FrancoArab Encounters, Beirut, American University of Beirut and Syracuse, New York, Syracuse University Press, p. 201-228. 
—, 1996b, «La Femme Arabe: Women and Sexuality in France's North African Empire », in Sonbol Amira El-Azhary (ed.), Women, the Family, and Divorce Laws in Islamic History, Syracuse, Syracuse University Press, p. 52-63.

—, 1998, "Islam, Gender, and Identities in the Making of French Algeria, 18301962 », in Clancy-Smith Julia \& Frances GoudA (eds), Domesticating the Empire. Race, Gender and Family Life in French and Dutch Colonialism, Charlottesville/Londres, University Press of Virginia, p. 154-173.

—, 2000, «L'École Rue du Pacha, Tunis : l'enseignement de la femme arabe et 'la Plus Grande France' (1900-1914) », in Leora AUSLANDER \& Michèle ZANCARINIFOURnel (dir.), «Le genre de la nation », CLIO. HFS, 12, p. 33-55.

—, 2007, «L'éducation des jeunes filles musulmanes en Tunisie: missionnaires religieux et laïques ", in Florence ROCHEFORT (dir.), Le pouvoir du genre : laïcités et religions 1905-2005, Toulouse, PUM, coll. «Le Temps du genre », p. 127-143.

—, 2010, Mediterraneans: North Africa and Europe in an Age of Migration, c. 1800-1900, Berkeley, University of California Press.

Clancy-Smith Julia \& Frances Gouda (eds), 1998, Domesticating the Empire. Race, Gender and Family Life in French and Dutch Colonialism, Charlottesville/Londres, University Press of Virginia.

Coco Carla, 2002, Harem, l'Orient amoureux, Paris, Éditions Place des Victoires.

CoDACCiOni Vanessa, 2010a, «Dépolitisation du genre et des questions sexuelles dans un procès politique en contexte colonial: le viol, le procès et l'affaire Djamila Boupacha (1960-1962)», Nouvelles Questions Féministes, 29 (version internet).

—, 2010b, «Quand les mouvements féministes font (avec) la loi : les lois du genre (2) », Nouvelles Questions Féministes, 29/1, p. 32-45.

Colonna Fanny \& Christelle TARAud, 2007, «La minorité européenne d'Algérie (1830-1962): inégalités entre “nationalités", résistances à la francisation et conséquences sur les relations avec la majorité musulmane», http://ensweb3.ens-lsh.fr/colloques/france-algerie/.

COLIN Joël, 1998, L'Enfant endormi dans le ventre de sa mère. Étude ethnologique et juridique d'une croyance au Maghreb, Perpignan, Presses universitaires de Perpignan.

CORBIN Alain, 2007, préface de Régis REVENIN (dir.), Hommes et masculinités de 1789 à nos jours, Paris, Autrement.

Croutier Lytle Alev, 1990, Harems : le monde derrière le voile, Paris, Belfond.

CuRTIS Sarah A., 2006, «Émilie de Vialar and the Religious Reconquest of Algeria », French Historical Studies, 29/3, p. 261-292. 
—, 2010, Civilizing Habits: Women Missionaries and the Revival of French Empire, Oxford, Oxford University Press.

DAKHLIA Jocelyne, 1999, "Entrées dérobées : l'historiographie du harem », in Agnès FINE \& Claudine LeDUC (dir.), «Femmes du Maghreb », CLIO. HFS, 9, p. 37-55.

—, 2000, "L'empire du favori », Les Cahiers du Centre de Recherches Historiques, 24, http://ccrh.revues.org/index1922.html.

—, 2007a, "Harem: ce que les femmes, recluses, font entre elles », in Christiane KLAPISCH-Zuber \& Florence Rochefort (dir.), "Clôtures », Clio. HFS, 26, p. 61-88.

—, 2007b, "Homoérotismes et trames historiographiques du monde islamique », Annales. HSS, 62/5, p. 1097-1120.

Damousi Joy, 1997, Depraved and Disorderly: Female Convicts, Sexuality and Gender in Colonial Australia, Cambridge, Cambridge University Press.

DAOuD Zakya, 1994, Féminisme et politique au Maghreb, Paris, Éditions Maisonneuve et Larose.

Decraene Philippe \& François Zuccareldi, 1994, Grands Sahariens à la découverte du " désert des déserts », Paris, Denoël.

DejEux Jean, 1984, "Elissa Rhaïs, conteuse algérienne (1876-1940)», Revue de l'Occident musulman et de la Méditerranée, 37, p. 47-79.

Delacour Marie-Odile \& Jean-René Huleu, 2008, Le voyage soufi d'Isabelle Eberhardt, Paris, Losfeld.

Delanoë Nelcya, 1999, La femme de Mazagan, Paris, Seghers.

—, 2002, Poussières d'Empire, Paris, PUF.

Del Plato Joan, 2002, Multiple Wives, Multiple Pleasures: Representing the Harem, 18001875, Vancouver, Fairleigh Dickinson University.

DORE-AudiBERT Andrée, 1995, Des Françaises d'Algérie dans la guerre de libération: des oubliées de l'bistoire, Paris, Karthala.

Dore-Audibert Andrée \& Annie Morzelle, 1997, Vivre en Algérie : des Françaises parlent : enquêtes, 1989-1995, Paris, Karthala.

Druot-Bouche Anne-Liz, 2001, "Genre, voyage et colonies : Une lecture des récits de voyage de femmes en Afrique du Nord, 1830-1930», mémoire de DEA, Université Marc Bloch.

DUTERTRE Anne, 1866, Voyage de Vernont-sur-Orme à Constantine sur l'Oued Rummel, Sétif, Bougie et Alger, par une femme, Caen, Imprimerie de Hommais.

Eaubonne (d') Françoise, 1985, Louise Michel la Canaque, Paris, Éditions Encre.

—, 1999 [1968], Vie d'Isabelle Eberhardt, Paris, Flammarion, J'ai lu. 
EICHNER Carolyn J., 2009, «La Citoyenne in the World: Hubertine Auclert and Feminist Imperialism », French Historical Studies, 32, p. 63-84.

EINAUDI Jean-Luc, 1994, Un rêve algérien : Histoire de Lisette Vincent, une femme d'Algérie, Paris, Dagorno.

El RouAyHeB Khaled, 2005, Before homosexuality in the Arabic-islamic world, 1500-1800, Chicago, University of Chicago Press.

ENNAJI Mohammed, 1994, Soldats, domestiques et concubines. L'esclavage au Maroc au XIXe siècle, Paris, Balland.

ERNOT Isabelle, 2008, "Le regard colonialiste de Madame Voisins d'Ambre », in Rebecca Rogers \& Françoise ThÉBAud (dir.), "Voyageuses », CLIO. HFS, 28, p. 185-193.

FAVRE Lucienne, 1930, Orientale, Paris, Grasset.

—, 1933, Tout l'inconnu de la casbah d'Alger, Alger, Baconnier.

FERHATI Barkahoum, 2003, «La danseuse prostituée dite "Ouled Nail”, entre mythe et réalité (1830-1962). Des rapports sociaux et des pratiques concrètes », in Christine BARD \& Christelle TARAud (dir.), "ProstituÉes », Clio. HFS, 17, Toulouse, PUM, p. 101-113.

Feyguine ABAdie Hélène, 1905, De l'assistance médicale des femmes indigènes en Algérie, Montpellier, Imprimerie Delord-Boehm et Martial.

FINE Agnès \& Claudine LeduC (dir.), 1999, «Femmes du Maghreb », CLIO. HFS, 9, Toulouse, Presses universitaires du Mirail.

FINLAYSON Ian, 1993, Tangier: City of the Dream, Londres, Flamingo.

FLeTCHER Simpson Yaël, 1998, " "Irresistible Seductions": Gendered Representations of Colonial Algeria around 1930 », in Julia CLANCY-SMITH \& Frances GOUDA (eds), Domesticating the Empire. Race, Gender and Family Life in French and Dutch Colonialism, Charlottesville \& Londres, University Press of Virginia, p. 193-210.

FLANDRIN Jean-Louis, 1981, Le sexe et l'Occident: évolution des attitudes et des comportements, Paris, Le Seuil.

Foche-Duval Monique, 2007, Madame S.A.S. : Femme d'officier : Algérie 1957-1962, Paris, François de Guibert Éditeur.

GAFSIA Nawel, 2008, L'invention coloniale du mariage musulman: le cas tunisien, Paris, LGDJ-Lextenso éd.

GARANGER Marc, 1982, Femmes algériennes 1960, Paris, Contrejour.

GAUDRY Mathéa, 1929, La femme chaonia de l'Aurès, Paris, Geuthner. 
—, 1961, La société féminine du Djebel Amour et du Ksel. Étude de sociologie rurale nordafricaine, Paris, Société algérienne d'impressions diverses.

Girardet Raoul, 1972, L'idée coloniale en France 1871-1962, Paris, La Table Ronde.

Girault Augustine (pseudonyme Anne Gaël), 1860, Souvenirs d'Algérie : quelques idées pratiques sur son défrichement, son assainissement, sa colonisation, Paris, Imprimerie de L. Guérin et Cie.

- 1868, La femme médecin, sa raison d'être, au point de vue du droit, de la morale et de l'bumanité, Paris, Le Dentu.

—, 1881, En Algérie, Paris, Librairie Centrale des Publications Populaires.

Goichon Anne-Marie, 1927, La vie féminine au M'zab, 2 tomes, Paris, Geuthner.

Goutalier Régine, 1989, «Les États généraux du Féminisme à l'exposition coloniale 30/31 mai 1931 », Revue d'Histoire Moderne et Contemporaine, p. 266-254.

GrosRichard Alain, 1979, Structure du sérail : la fiction du despotisme asiatique dans l'Occident classique, Paris, Le Seuil.

GuiARD Claudine, 2008, «La place des Européennes dans la colonisation de l'Algérie (1830-1939). Place impartie, place occupée», in Amandine LAuro (dir.), "Colonialismes », Sextant, Revue du groupe interdisciplinaire sur les femmes et le genre, 25, Bruxelles, p. 130-140.

—, 2009, Des Européennes en situation coloniale. Algérie 1830-1939, Aix-en-Provence, Éditions de l'Université de Provence.

—, 2011, «La colonisation au miroir de la presse féminine et féministe dans l'Algérie des années 1930 », in Philippe Mezzasalma \& Christelle Taraud (dir.), « Presses et Colonies », Revue de la Bibliothèque nationale de France, Paris (sous presse).

HA Marie-Paule, 2005, «'La Femme française aux colonies': Promoting Colonial Female Emigration at the Turn of the Century », French Colonial History, 6. (version internet sur le site de $\mathrm{FCH}$ ).

Halimi Gisèle \& Simone de BEAuvoir, 1962, Djamila Boupacha, Paris, Gallimard.

Hanoum Leila, 2000, Le harem impérial an XIXe siècle, Bruxelles, Complexe.

Hart Kingsmill Ursula, 1986, Two Ladies of Colonial Algeria: The Lives and Times of Aurélie Picard and Isabelle Eberhardt, Ohio, Ohio University, Centre for International Studies.

Hataway Jane, 1992, "The Role of the Kizlar Agasi in 17th and 18 th Century Egypt », Studia Islamica, 75, p. 141-158.

—, 1994, "The Wealth and Influence of an Exiled Ottoman Eunuch in Egypt: The Waqf Inventory of Abbas Agha », Journal of the Economic and Social history of the Orient, 37/4, p. 293-317. 
—, 2005, Beshir Agha: Chief Eunuch of the Ottoman Imperial Harem, London, Oneworld Publications.

HAYES Jarrod, 2000, Queer Nations: Marginal Sexualities in the Maghreb, Chicago, Chicago University Press.

Hefferman M.J., 1989, "The Parisian Poor and the Colonisation of Algeria during the Second Republic », French History, 3/4, p. 377-403.

Helie-LuCAs Anissa, 1988, «Les institutrices européennes laïques en Algérie (18741949) », mémoire de DEA, Université d'Aix-en-Provence.

Hodgson Barbara, 2002, Les Aventurières, XVIIe-XIXe siècle. Récits de femmes voyageuses, Paris, Le Seuil.

- 2005, Dreaming on the East: Western Women and the Exotic Allure of the Orient, Vancouver, Greystone Books.

Huart (d') Annabelle \& Nadia Tazi, 1980, Harems, Paris, Éditions du Chêne.

Hunt Rose Nancy, LiU Tessie \& Jean QuATAerT (eds), 1997, Gendered Colonialisms in African History, Oxford, Blackwell.

Ighilahriz Louisette, 2001, Algérienne, récit recueilli par Anne Nivat, Paris, Fayard.

JAuffret Jean-Charles \& Charles-Robert Ageron (dir.), 2003, Des hommes et des femmes en guerre d'Algérie, Paris, Autrement.

JAUNEAU Elodie, 2008, «L'engagement des femmes dans l'armée française durant la guerre d'Algérie (1954-1962)», in Amandine LAURO (dir.), "Colonialismes », Sextant, Revue du groupe interdisciplinaire sur les femmes et le genre, 25, Bruxelles, Éditions de l'Université de Bruxelles, p. 71-85.

JouIN Jeanne, 1932, Iconographie de la mariée citadine dans l'islam nord-africain, Paris, P. Geuthner.

Julien Charles-André, 1931, Histoire de l'Afrique du Nord, Paris, Payot.

KALIFA Dominique, 2009, Biribi : les bagnes coloniaux de l'armée française, Paris, Perrin.

Kassis Annpôl, 2010, Les disparues de l'Amphitrite. Des femmes déportées en Nouvelle-Galles du Sud, Paris, Janus.

KazDaghli Habib, 2000, «L'éducation de la femme dans la Tunisie colonisée : représentations et moyens mis en œuvre », in Dalenda LARGuÈCHE (dir.), Histoire des femmes au Maghreb: culture matérielle et vie quotidienne, Tunis, Centre de publications universitaires.

Kerrou Mohamed \& Moncef M'Halla, 1993, «La prostitution dans la médina de Tunis aux XIXe et XX $x^{\mathrm{e}}$ siècles ", in Fanny COLONNA (dir.), Ëtre marginal au Maghreb, Paris, CNRS Éditions, p. 201-221. 
KIMBLE Sara L., 2006, «Emancipation through secularization: French feminist views of Muslim women's condition in interwar Algeria ", French colonial bistory, 7, p. 109-128.

KNIBIEHLER Yvonne, 1993, «L'instruction des filles au Maroc pendant le protectorat (1912-1956)», Sextant, 1, p. 99-112.

KnibielHer Yvonne \& Régine Goutalier, 1985, La femme aux temps des colonies, Paris, Stock.

KrakOVITCH Odile, 1998 [1990], Les femmes bagnardes, Paris, Olivier Orban.

Lallaoui Medhi, 1999, Kabyles du Pacifique, Paris, Alternatives.

LALAMI Feriel, 2008, «L'enjeu du statut des femmes durant la période coloniale en Algérie », Nouvelles questions féministes, 27/3, p. 16-27.

LaOust-ChantréAux Germaine, 1990, Kabylie côté femmes : la vie féminine à Aït Hichem, 1937-1939: notes d'ethnographie, Aix-en-Provence, Édisud.

LAPEYRE Françoise, 2008, Le roman des voyageuses françaises (1800-1900), Paris, Payot.

LARBI SNOUSSI Mohamed, 2000, «La prostitution en Tunisie au temps de la colonisation », in Jacques Alexandropoulos \& Patrick CABAnel (dir.), La Tunisie Mosä̈que, Toulouse, Presses Universitaires du Mirail, p. 389-413.

LARguÈche Dalenda \& Abdelhamid, 1992, Marginales en terre d'Islam, Tunis, Cérès Productions.

LARGUÈCHE Abdelhamid, 2000, Les ombres de Tunis, pauvres, marginaux et minorités aux XVIII et XIXe siècles, Paris, Arcanteres.

LARGuÈche Dalenda (dir.), 2000, Histoire des femmes au Maghreb. Culture matérielle et vie quotidienne, Tunis, Centre de publications universitaires.

Lauro Amandine (dir.), 2008, «Colonialismes", Sextant, Revue du groupe interdisciplinaire sur les femmes et le genre, 25, Bruxelles, Éditions de l'Université de Bruxelles

LAZReg Marnia, 1994, The Eloquence of Silence: Algerian Women in Question, New York \& Londres, Routledge.

Lecour-Grandmaison Olivier, 2005, Coloniser, Exterminer. De la guerre et de l'État colonial, Paris, Fayard.

LEGRAND-FALCO Marcelle, 1931, «Les états généraux du féminisme à l'exposition coloniale de 1931 », La Volonté, 3 avril.

LE NAOUR Jean-Yves, 2004, La Honte noire. L'Allemagne et les troupes coloniales françaises (1914-1945), Paris, Hachette-Littératures.

—, 2006, «La "honte noire”. La haine raciale des Allemands à l'encontre des troupes coloniales de l'armée française (1914-1940)», Quasimodo, 8, p. 245-254. 
LenZIni José, 1998, Aurélie Picard, princesse Tidjani, Paris, La Renaissance.

LeVINE Philippa, 2004, Gender and Empire, Oxford, Oxford University Press.

LEWIS Reina, 1996, Gendering Orientalism: Race, Femininity and Representation, New York \& Londres, Routledge.

LORCIN Patricia, 1995, Imperial Identities: Stereotyping, Prejudice and Race in Colonial Algeria, Londres \& New York, St Martin's Press.

—, 2002, "Women, Gender and Nation in Colonial Novels of Interwar Algeria », Historical Reflections/Réflexions historiques, 28, p. 163-184.

- 2003, "Sex, Gender, and Race in the Colonial Novels of Elissa Rhaïs and Lucienne Favre », in Sue Peabody \& Tyler Stovall (eds), The Color of Liberty: Histories of Race in France, Durham, Duke University Press, p. 108-130.

—, 2004, "Mediating Gender, Mediating Race: Women writers in colonial Algeria », Culture, Theory and Critique, 45/1, p. 45-61.

Mabon Armelle, 2010, Prisonniers de guerre «indigènes ». Visages oubliés de la France occupée, Paris, La Découverte.

MARZOUKI Ilhem, 1993, Le Mouvement des femmes en Tunisie au XX'e siècle : féminisme et politique, Paris, Maisonneuve et Larose.

Massad Joseph A., 2007, Desiring Arabs, Chicago, University of Chicago Press.

Mauss-Copeaux Claire, 1998, Appelés en Algérie. La parole confisquée, Paris, HachetteLittératures, coll. « Pluriel ».

—, 2003, À travers le viseur, Algérie 1955-1962, Lyon, Aedelsa.

MCINTYRe Perry, 2010, Free Passage: The Reunion of Irish Convicts and Their Families in Australia 1788-1852, Irish Academy Press Ltd.

Melman Billie, 1995, Women's Orients: English Women and the Middle East, 1718-1918: Sexuality, Religion and Work, Basingstoke, Macmillan.

Mernissi Fatima, 1983, Sexe, idéologie, islam, Paris, Tierce.

—, 1987, Le harem politique. Le prophète et les femmes, Paris, Albin Michel.

—, 1990, Sultanes oubliées. Femmes chef d'État en islam, Paris, Albin Michel.

—, 2001, Le harem et l'Occident, Paris, Albin Michel.

MESSAADI Sakina, 1990, Les romancières coloniales et la femme colonisée : contribution à une étude de la littérature coloniale en Algérie, Alger, Entreprise Nationale du Livre.

—, 2002, Nos sæurs musulmanes ou le mythe féministe, civilisateur, évangélisateur du messianisme colonialiste dans l'Algérie colonisée, Alger, Houma.

MiCHAEL Gil, 2005, «L'effacement de la cantinière ou la virilisation de l'armée française au XIXe siècle », Revue d'bistoire du XIXe siècle, 30, p. 21-43. 
MiCHel Louise, 1885, Légendes et chants de gestes canaques, Paris, Keva et $\mathrm{C}^{\circ}$ Editeurs.

Midgley Clare, 1998, Gender and Imperialism, Manchester, Manchester University Press.

Mills Sarah, 1993, Discourses of Difference: An Analysis of Women's Travel Writing and Colonialism, New York, Routledge.

Mink Paule, 1886, «La femme en Algérie », Le coup de feu, 5, janvier, p. 12-14.

MoniCAT Bénédicte, 1996, Itinéraire de l'écriture au féminin. Voyageuses du XIXe siècle, Amsterdam/Atlanta, Rodopi.

—, 2003, «L'Algérie des voyageuses », in Emanuele KANCEFF (dir.), Stendhal, l'Italie, le voyage. Mélanges offerts à $V$. Del Litto, Moncalieri, CIRVI, p. 377-388.

Montagu Mary (Lady), 2001, L'Islam au péril des femmes. Une Anglaise en Turquie au XVIII siècle, Paris, La Découverte.

M'RABet Fadela, 1967, Les Algériennes, Paris, Maspero.

Nochlin Linda, 1995, "L'Orient imaginaire », in Les politiques de la vision. Arts, société et politique, Paris, Éditions Jacqueline Chambon.

Noirfontaine de Pauline, 1856, Algérie. Un regard écrit, Le Havre, Lemale.

Nouvel Suzanne, 1919, Nomades et sédentaires au Maroc, préface d'Augustin BERNARD, Paris, E. Larose.

OTMEzGuine Jacques, 1993, Le secret d'Elissa Rhaïs, téléfilm.

OxLEY Deborah, 1996, Convict Maids: The Forced Migration of Women to Australia, Cambridge, Cambridge University Press.

Palmier-Chatelain Marie-Élise \& Pauline Lavagne D’Ortigue (dir.), 2002, L'Orient des femmes, Lyon, ENS Éditions.

Patane Vincenzo, 2006, "L'homosexualité au Moyen-Orient et en Afrique du Nord ", in Robert ALDRICH (dir.), Une histoire de l'homosexualité, Paris, Éditions du Seuil, p. 271-301.

PIERCE Leslie, 1993, The Imperial Harem. Women and Sovereignty in the Ottoman Empire, Oxford, Oxford University Press.

PLISSON Odile, 1972, J'étais assistante sociale avec les combattants d'Algérie, Paris, La Pensée Universelle.

RABet M’ Fadela, 1967, Les Algériennes, Paris, Maspero.

REBREYEND Anne-Claire, 2005, "Comment écrire l'histoire des sexualités au XXe siècle? ", in Sylvie CHAPERON \& Agnès FINE (dir.), "Utopies sexuelles », CLIO. HFS, 22, p. 185-209.

RENUCCI Françoise, 1983, Souvenirs de femmes au temps des colonies, Paris, Balland. 
ReVeillaud De Lens Aline, 1922, Derrière les vieux murs en ruines. Roman marocain, Paris, Calmann-Lévy.

—, 1925, Pratiques des harems marocains : sorcellerie, médecine, beauté, Paris, P. Geuthner.

—, 2006, Journal 1902-1924 : l'amour, je le supplie de m'épargner, Paris, La cause des livres.

RIVET Daniel, 1992, «Le fait colonial et nous : histoire d'un éloignement », Vingtième siècle. Revue d'histoire, 33, janvier-mars, p. 127-138.

RIVIÈRE Thérèse, 1995, Aurès/Algérie: photographies 1935-1936, Paris, Éditions de l'EHESS.

ROBERTS Mary, 2008, Intimate Outsiders: The Harem in Ottoman and Orientalist Art and Travel Literature, Durham, Duke University Press.

RoBinson Jane, 1999, Parrot Pie for Breakefast: An Anthology of Women Pioneers, Oxford, Oxford Paperbacks.

RoBInson-Dunn Diane, 2006, The Harem, Slavery and British Imperial Culture: AngloMuslim Relations in the Late Nineteenth Century, Manchester, Manchester University Press.

ROGERS Rebecca, 2008, «Décrypter le regard national: voyageuses anglaises et françaises en Algérie », in Nicolas Bourguinat (dir.), Le voyage au féminin. Aspects bistoriques et littéraires (XVIII -XX ${ }^{e}$ siècles), Strasbourg, Presses universitaires de Strasbourg, p. 89-106.

—, 2009, «Telling Stories about the Colonies: English and French Women in Algeria in the 19th century », Gender and History, avril, p. 39-59.

Rogers Rebecca \& Françoise THÉBAud (dir), 2008, «Éditorial» de CLIO. HFS, 28, «Voyageuses », Toulouse, PUM, p. 5-16.

Roscoe Will \& Stephen O. Murray (eds), 1997, Islamic Homosexualities: Culture, History, and Literature, New York, New York University Press.

RuDE Fernand, 1981, Bagnes d'Afrique : trois transportés en Algérie après le coup d'État du 2 décembre 1851, Paris, Maspero.

RusCIO Alain, 1996, Amours coloniales, aventures et fantasmes érotiques de Claire de Duras à Georges Simenon, Bruxelles, Complexe.

SAADA Emmanuelle, 2007, Les enfants de la colonie. Les métis de l'Empire français entre sujétion et citoyenneté, Paris, La Découverte.

SAI Fatima Zohra, 1984, Mouvement national et question féminine : des origines à la veille de la guerre de libération nationale, Oran, CRIDSSH.

SAmbron Diane, 2007, Femmes musulmanes. Guerre d'Algérie (1954-1962), Paris, Autrement.

—, 2009, Les Femmes algériennes pendant la colonisation, Paris, Riveneuve. 
SCHREIER Joshua Winter, 2007, «Napoléon's Long Shadow: Morality, Civilization, and Jews in France and Algeria, 1808-1870 », French Historical Studies, 30/1, p. 77103.

SChmitT Arno \& Jehoeda Sofer, 1992, Sexuality and Eroticism Among Males in Moslem Societies, Harrington Park Press Inc.

SEFERDJELI Ryme, 2004, "French Reforms and Muslim Women's emancipation during the Algerian War », The Journal of North African Studies, 9/4, p. 19-61.

-, 2005, "The French Army and Muslim Women during the Algerian War", Hawwa-Journal of Women in the Middle East and Islamic World, 3/1, p. 40-78.

—, 2007, «Les femmes dans l'ALN : le mariage et/ou l'action ? ", Colloque pour une histoire critique et citoyenne, le cas de l'histoire franco-algérienne, 20-22 juin 2006, Lyon, ENS-LSH, http://ens-web3.ens-lsh.fr/colloques/francealgerie/communication.php3?id_article $=261$

SELzer Anita, 1994, Educating Women in Australia: From the Convict Era to the 1920s, Cambridge, Cambridge University Press.

SHEPPARD Todd, 2004, "La "bataille du voile" pendant la guerre d'Algérie ", in Nordmann Charlotte (ed.), Le foulard islamique en questions, Paris, Éditions Amsterdam, p. 134-141.

—, 2008, 1962. Comment l'indépendance algérienne a transformé la France, Paris, Payot.

SLAVIN David Henry, 2001, Colonial Cinema and Imperial France, 1919-1939: White Blind Spots, Male Fantasies, Settler Myths, Baltimore, John Hopkins University.

SLIMANI DiRÈche Karima, 2004, Chrétiens de Kabylie (1873-1954). Une action missionnaire dans l'Algérie coloniale, Paris, Éditions Bouchène.

SOAZIC Lahuec, 2009, "Tentative d'assassinat d'Isabelle Eberhardt: un dossier judiciaire qui interroge », Cabiers de la Méditerranée, 78, p. 307-316.

SRAIEB Noureddine, 1999, "Islam, réformisme et condition féminine en Tunisie : Tahar Haddad (1898-1935) », in Agnès FINE \& Claudine LEDuC (dir.), « Femmes du Maghreb », CLIO. HFS, 9, Toulouse, PUM, p. 75-92.

STOLER Ann Laura, 1989, "Making Empire Respectable: The Politics of Race and Sexual Morality in 20th Century Colonial Cultures ", American Ethnologist, 16/4, novembre, p. 634-660.

- 1995, Race and the Education of Desire: Foucault's History of Sexuality and the Colonial Order of Things, Durham, Duke University Press.

Strobel Margaret, 1993, "Gender, Sex and Empire », in Michael AdAS (dir.), Islamic and European Expansion: the Forging of a Global Order, Philadelphie, Temple University Press, p. 345-375. 
SwISS Deborah J, 2010, The Tin Ticket: The Heroic Journey of Australia's Convict Women, Berkeley Publishing Corporation.

TAвEт Paul, 1982, Elissa Rhaïs, Paris, Grasset.

TAÏEB Edith, 2006, "Coloniser and colonised in Hubertine Auclert's writings on Algeria », in Diana Holmes \& Carrie TARR (dir.), "A Belle Epoque”? Women in French society and culture: 1890-1914, New York, Berghann Books, p. 271-281.

TARAud Christelle, 2003a, La prostitution coloniale. Algérie, Tunisie, Maroc, 1830-1962, Paris, Payot.

—, 2003b, Femmes orientales dans la photographie coloniale, 1860-1910, Paris, Albin Michel.

—, 2004, "Angélique et l'Orient: une certaine vision de l'altérité ? ", in Nicole Beaurain, Larry Portis, Christiane Passevant \& Christelle Taraud (dir.), « Le cinéma populaire et ses idéologies ", L’Homme et la société, 154, octobre-décembre, L'Harmattan, p. 9-29.

—, 2007, «Les femmes marocaines au sortir du Protectorat : entre tradition et émancipation ", in Mohammed KENBIB (dir.), Actes du colloque international $D u$ Protectorat à l'indépendance. Problématique du temps présent, Rabat, Éditions de l'Université Mohammed V, p. 115-125.

—, 2008a, «Genre, sexualité et colonisation. La colonisation française au Maghreb », in Amandine LAuro (dir.), "Colonialismes", Sextant. Revue du groupe interdisciplinaire sur les femmes et le genre, 25, Bruxelles, p. 117-127.

—, 2008b, «Les yaouleds : entre marginalisation sociale et sédition politique. Retour sur une catégorie hybride de la casbah d'Alger dans les années 1930-1960 », Revue d'histoire de l'enfance « irrégulière » (RHEI), 10, octobre, p. 59-74.

- 2008c, "Genre, classe et "race" en contexte colonial et post-colonial : une approche par la mixité sexuelle », in Pascale BOnNEMÈRE \& Irène THÉRY (dir.), Ce que le genre fait aux personnes, Paris, Éditions de l'EHESS, coll. "Enquête », p. 157-171.

—, 2009a, «Les bagnes de l'Empire au féminin: où comment déporter les opposantes politiques de la métropole dans les colonies françaises au XIX ${ }^{\mathrm{e}}$ siècle ", Sextant. Revue du groupe interdisciplinaire sur les femmes et le genre, 25, Bruxelles, p. $17-25$.

—, 2009b, "Le rêve masculin de femmes dominées et soumises », in Driss EL Yazami, Yvan Gastaut \& Naïma YAHI (dir.), Générations. Un siècle d'bistoire culturelle des Maghrébins en France, Paris, Gallimard/Génériques CNHI, p. 63-68.

_, 2009c, « Prendre femme dans les colonies », L'Histoire, 340, mars, p. 70-75. 
—, 2011, "La virilité en situation coloniale et post-coloniale », in Alain CORBIN (dir.), Histoire de la virilité XIX ${ }^{e}$ siècle/Première Guerre mondiale, t. 2 ; et Jean-Jacques Courtine (dir.), Histoire de la virilité $X X^{e} / X X I^{e}$ siècles, t. 3, Paris, Seuil.

—, Sexes et colonies. Virilité, " homosexualité » et «tourisme sexuel » au Maghreb (XIX ${ }^{e}$ et XX ${ }^{e}$ siècles), Paris, Payot (à paraître).

Temine Ben Blili Leilla, 1999, Histoires de familles. Mariages, répudiations et vie quotidienne à Tunis, 1875-1930, Tunis, Script Éditions.

THORNTON Lynne, 1994, La femme dans la peinture orientaliste, Paris, ACR Éditions.

Tillion Germaine, 1966, Le harem et les cousins, Paris, Le Seuil.

TURIN Yvonne, 1971, Affrontements culturels dans l'Algérie coloniale: École, médecine, religion, 1830-1880, Paris, Maspero.

Verges Jacques \& Georges Arnaud, 1957, Pour Djamila Bouchired, Paris, Éditions de Minuit.

WiarT Carole, 1997, «Hubertine Auclert, une féministe en Algérie (1888-1892) », mémoire de maîtrise, Université Paris 8.

Wood Diane, 2009, «Les voyageuses françaises en Algérie. Une analyse des récits de voyage entre 1848 et 1856 », mémoire de master, sous la direction de Christelle Taraud, Paris, NYU.

YEE Jennifer, 2000, Clichés de la femme exotique : un regard sur la littérature coloniale française entre 1871 et 1914, Paris, L'Harmattan.

Yeyenoglu Meyda, 1998, Colonial fantaisies: toward a feminist reading of Orientalism, Cambridge, Cambridge University Press.

Zagnoli Nello \& Claude BréteAu, 1981, «Le statut de la femme dans deux communautés rurales méditerranéennes : la Calabre et le nord-est constantinois », Les temps modernes, 418, mai, p. 1954-2007.

ZeYs Mathilde, 1908, Une Française an Maroc, Paris, Hachette.

Zemmour Nathalie, 1992, "Les naissances illégitimes en Algérie au XIX et au XX ${ }^{\mathrm{e}}$ siècles », mémoire de DEA, Université Paris I - Panthéon Sorbonne.

Zerdoumi Nefissa, 1970, Enfants d'bier, enfants d'aujourd'bui. L'éducation de l'enfant en milieu traditionnel algérien, Paris, Maspero. 